\title{
Beschimpfen, Gerüchte- verbreitung und Verunglimpfung im virtuellen Raum: Messmodell zu Einstellungen gegenüber Cybermobbing bei Schülerinnen und Schülern
}

\section{Horst Biedermann, Arvid Nagel und Fritz Oser}

Cybermobbing stellt ein nicht zu unterschätzendes Problem der heutigen Sozialen Medien dar. In diesem Beitrag interessiert, (1) ob sich Einstellungen gegenüber Cybermobbing empirisch erfassen lassen und (2) welche Einstellungen Schülerinnen und Schüler gegenüber Cybermobbing haben. ie Stichproben der vorliegenden Untersuchung entstammen der IEA-Ländervergleichsstudie ICILS (Schweizer Modul) und der Studie Lebenswelten Ostschweizer Jugendlicher (LeOJ). Das Alter der befragten Schülerinnen und Schüler liegt zwischen 12 und 16 Jahren. Zur Erfassung der Einstellung gegenüber Cybermobbing wurde ein Instrument neu entwickelt, welches hier vorgestellt wird. Die beiden Datengrundlagen bestätigen ein theoretisch begründetes g-Faktormodell. Die Ergebnisse zeigen, dass die Schülerinnen und Schüler gegenüber Cybermobbing in deutlicher Mehrheit negativ eingestellt sind.

\section{Einstellungen gegenüber Cybermobbing}

Einstellungen zu verletzendem Handeln, wie es das Cybermobbing darstellt, sind bis jetzt wenig untersucht worden. Wenn wir dies hier tun, so im Bewusstsein, dass eine Einstellung ein auf Erfahrungen basierendes bewertendes Urteil von Dingen, Zuständen, aber auch von Personen und sozialen Gegebenheiten darstellt. Einstellungen beruhen immer auf kognitiven, affektiven und verhaltensbezogenen Informationsverarbeitungen. Meistens werden diese drei Dimensionen ganzheitlich und nicht getrennt voneinander erschlossen. In der Literatur findet man zudem, dass Einstellungen und Überzeugungen häufig synonym verwendet werden, was nach Eagly und Chaiken (1993) eine Reduktion auf die kognitive Dimension bedeutet (z.B. Fazio \& Petty, 2007; Haddock \& Maio, 2014; Maio \& Haddock, 2010). Man kann davon ausgehen, dass Einstellungen Verhalten vorherzusagen vermögen - wie es auch in der Theorie des geplanten Verhaltens (Ajzen, 1985, 1991) vertreten wird. Diese Voraussagekraft fällt insbesondere dann eng(er) aus, wenn die folgenden Bedingungen erfüllt sind: (1) Korrespondenz zwischen Einstellungs- und Verhaltensmassen 
(z.B. wenn eindeutige und einfache Operationalisierungen gegeben sind), (2) einfach umzusetzender Verhaltensbereich (z.B. wenn ein Verhalten einfach einzulösen ist, wie die Stimmabgabe für eine politische Partei), (3) Hervortreten von stärker als normal ausgebildeter Einstellung (z.B. wenn häufig bestätigte negative Erfahrungen begleitet mit starken Emotionen gegenüber Menschen aus anderen Kulturen gemacht wurden), und (4) spezifische Persönlichkeitsmerkmale (z.B. wenn eine geringe Selbstüberwachung vorhanden ist) (Haddock \& Maio, 2014, S. 219ff.).

In diesem Beitrag geht es spezifisch um Einstellungen gegenüber Cybermobbing. Unter Cybermobbing oder Cyberbullying wird die Belästigung, Beleidigung, Bedrohung oder Blossstellung von Personen mittels neuer Kommunikationsmedien (wie Smartphones, E-Mails, Websites, Foren, Chats und Communities) verstanden (u.a. Katzer, 2014; McQuade, Colt \& Meyer, 2009). Es handelt sich also um eine Nutzung elektronischer Kommunikationsmittel auf eine Art und Weise, die gesellschaftlich nicht erwünscht ist, vor dem Hintergrund ethischer Normen moralisch fragwürdig ist, unter sozialen Standards diskriminierend wirkt und vor dem Gesetz teilweise auch verboten ist. Cybermobbing ist somit die Aktualisierung einer negativen Einstellung, wie wir es nennen. Es ist damit ein Verhältnis zu einem potentiell schädigenden Tun gemeint (ohne sich in das Leid der Betroffenen zu versetzen, wobei die Täterschaft zudem meistens unerkannt bleiben möchte und auch glaubt, ungeschoren davon zu kommen). In diesem Aufsatz geht es um diese negative Einstellung, die - das kann vorausgesetzt werden - insbesondere dann stark auf die Opfer wirkt, wenn eine Mehrzahl an Menschen die Handlungen der Täterschaft (weitgehend) verfolgen und stillschweigend zur Kenntnis nehmen (Bystander). Je weniger junge Menschen Cybermobbing bejahen, desto weniger negative Einstellung ist vorhanden. Trotzdem wissen wir noch wenig darüber, wie Jungen und Mädchen die Lust und den Drang bzw. eine Einstellung entwickeln, dieses Handeln zu aktualisieren (siehe dazu die Bestandesaufnahme von Petermann \& von Marées, 2013) - dass aber derartige Entwicklungen stattfinden, können wir (leider) regelmässig der Presse entnehmen, wobei sich die Opfer häufig selbst auch über die Entwicklungen überrascht zeigen: «Es kam einfach - und ich konnte nichts dagegen tun» (DIE ZEIT, 2013). ${ }^{1}$

\section{Cybermobbing bei Jugendlichen: theoretische Grundlagen und allgemeiner Forschungsstand}

Die Thematik Cybermobbing gewinnt sowohl im nationalen als auch im internationalen Kontext zunehmend an öffentlichem und wissenschaftlichem Interesse (z.B. Baier, Krenz \& Bergmann, 2016; Bergmann \& Baier, 2016; Erdur-Baker, 2010; Erentaité, Bergman \& Žukauskiené, 2012; Fenaughty \& Harré, 2013; Festl, 2015; Gradinger, Strohmeier \& Spiel, 2009; Gradinger, Yanagida, Stroh- 
meier \& Spiel, 2016; Langos, 2012; Petermann \& von Marées, 2013; Pieschl \& Porsch, 2014; Slonje \& Smith, 2008; Smith, Mahdavi, Carvalho, Fisher, Russell \& Tippett, 2008; Voskamp \& Kipker, 2013; Weinstein \& Selman, 2014), wobei - wie gesagt - der Blick auf die Einstellungen gegenüber Cybermobbing bis dato noch kaum eingenommen wird (Festl, 2016; Festl, Scharkow \& Quandt, 2013, 2015; Porsch \& Pieschl, 2014; Riebel, Jäger \& Fischer, 2009). Für den Forschungsstand ist es von Bedeutung, dass Cybermobbing mit dem - durch die Mediengeschichte - früheren Begriff des traditionellen Mobbings in Zusammenhang gebracht wird. Auch hier geht es um kommunikative Handlungen, die «gegen eine Person gerichtet sind (von einer oder mehreren anderen) und die sehr oft und über einen längeren Zeitraum hinaus vorkommen und damit die Beziehung zwischen Täterin bzw. Täter und Opfer kennzeichnen" (Leymann, 1993, S. 22; hierzu u.a. Schubarth, 2012; Teuschel, 2010; Wachs, Hess, Scheithauer \& Schubarth, 2016). Das Machtungleichgewicht zwischen Täterin bzw. Täter und Opfer, zeichnet sich dadurch aus, dass die ersteren dem tendenziell wehrlosen zweiten überlegen sind. Die Überlegenheit baut sich auf Formen wie beleidigender Beschimpfungen (Flaming), Belästigung (Harassment), Verunglimpfung und Verbreitung von Gerüchten (Denigration), betrügerischem Auftreten wie Übernahme von Online-Identitäten anderer Personen (Impersanotion), Verrat und Blossstellung (Trickery and Outing), Ausschluss (Exclusion), fortwährende Belästigung und Verfolgung ([Cyber-]Stalking) und offene Androhung von Gewalt ([Cyber-]Threats) auf (Willard, 2007). In jüngeren Diskussionen wird ergänzend dazu das sogenannte Happy Slapping erwähnt (Özöz, 2013). Bei dieser Form wird physische Gewalt mit Handys aufgezeichnet und verbreitet. Eine weitere Form ist die sexuelle Belästigung, bei der das Opfer ungewollt pornographische Inhalte erhält oder zu sexuellen Handlungen vor der Webcam genötigt wird. Diese Formen können sowohl einzeln auftreten, ineinander übergehen als auch simultan erscheinen. Inwieweit die Arten des traditionellen und virtuellen Mobbings in Beziehung stehen, analysierten Neuber, Künsting und Phieler (2014). Ihre Befunde zeigen, dass von den 12-19-Jährigen, die Cybermobbing vornahmen, auch die meisten an traditionellem Bullying bzw. Mobbing beteiligt waren (hierzu u.a. auch Kowalski, Morgan \& Limber, 2012; Wachs \& Wolf, 2011). Für diese Jugendlichen zeigen sich zudem Beziehungen zu Neurotizismus (positiv) und Verträglichkeit (negativ).

Während die Befundlage zu den Einstellungen gegenüber Cybermobbing noch rar ausfällt, kennzeichnet sie sich hinsichtlich der Erscheinungsformen (z.B. Kern, 2014; Perren et al., 2012), zu den Prävalenzen (z.B. Feierabend, Plankenhorn \& Rathgeb, 2016), der Begünstigungsfaktoren (z.B. Festl, Vogelgesang, Scharkow \& Quandt, 2017), der Ursachen (z.B. Perren \& Gutzwiller-Helfenfinger, 2012; Sticca, Perren, Ruggieri \& Alsaker, 2013), der Folgen (z.B. Waller, Willemse, Genner, Suter \& Süss, 2016) und der Präventionsmöglichkeiten (z.B. Slonje, Smith \& Frisén, 2013) von Cybermobbing durchaus 
reich. Insgesamt zeigt sich, dass die Form des Cybermobbings in heutiger Zeit ubiquitär ist und von allen jederzeit und ortsungebunden mit Zugang zu neuen Technologien weitgehend unkontrolliert vorgenommen werden kann. Petermann und von Marées (2013) schlussfolgern aus ihrer Forschungsübersicht, dass vor allem bei Jugendlichen zwischen 10 und 16 Jahren eine Zunahme dieses Phänomens festzustellen sei (sowohl in Gestalt von Täterschaft als auch von Opfererfahrungen).

Zu erwähnen gilt es aber auch die Rolle der dritten beteiligten Gruppe, nämlich jener der wissenden Zuschauenden und/oder Mitlesenden, welche aber dennoch nichts unternimmt. Dieses Mitverfolgen des Geschehens ist, wie oben erwähnt, eine Art Bystander-Effekt, welcher sich insbesondere dadurch auszeichnet, dass das Geschehen unaufhaltsame Verbreitung und damit (verborgene) Resonanz erfährt, was dem Ziel des Cybermobbings deutlich zudient. Denn dieser Effekt besagt, je mehr Personen mitbekommen, dass einer Drittperson Unrecht geschieht, desto kleiner ist die Wahrscheinlichkeit, dass diese beobachtenden Personen auch eingreifen. Gutzwiller (2015) sowie Perren und Gutzwiller-Helfenfinger (2012) haben diesen Effekt u.a. unter dem Gesichtspunkt des moral disengagement untersucht. Sowohl das Fehlen moralischer Werte als auch das Fehlen von Gewissensbissen sagt negatives Täterinnen- bzw. Täter- und Bystander-Verhalten voraus. Obwohl Bystander auf den ersten Blick «nichts tun", nehmen diese (gerade aufgrund des [Nicht-]Verhaltens) eine wichtige Funktion hinsichtlich des Ausmasses der Schädigung ein, insbesondere wenn es sich um Formen indirekten Cybermobbings handelt. Hier wird die schädigende Kommunikation nicht an das Opfer selbst adressiert, sondern es werden verletzende Aussagen über das Opfer an ein grösseres Publikum gerichtet. Die Auseinandersetzung wird damit also bewusst in die Öffentlichkeit getragen. Eine positive Einstellung gegenüber Cybermobbing zu haben meint somit, dass man ein derartiges Verhalten (allgemein oder unter bestimmten Bedingungen) als nicht verwerflich und womöglich sogar als zweckmässig oder gar rechtmässig bewertet.

In der Entwicklung unseres Testinstruments wählten wir einen allgemein cybermodalen Zugang zum interessierenden Phänomen der Einstellungen gegenüber Cybermobbing, d.h. wir unterscheiden nicht explizit zwischen den oben dargestellten Formen (Özöz, 2013; Willard, 2007). Die Itemformulierungen sind allgemein auf alle Arten der Beleidigung und des Beschimpfens, daher wird die Operationalisierung und Messmodelltestung eines Generalfaktors gewählt. Ebenfalls lassen wir den Zeitaspekt aussen vor, d.h. wir fragen nicht nach den Konsequenzen bzw. ob schädigende Inhalte im virtuellen Raum zurückgenommen werden können oder dort dauerhaft verbleiben und dadurch von selber wiederholt aufgerufen werden (können), wodurch es erst zu einer unkontrollierbaren Verbreitung kommen kann (z.B. Kindler, 2011). Zudem differenzieren wir auch nicht explizit nach den Dimensionen kognitiv, emotional und verhaltensbezogen, sondern implizieren auch diese in eine übergeordnete Operationalisierung - wobei es auch hier festzuhalten gilt, dass die Formulie- 
rungen primär die kognitive Dimension betreffen.

\section{Zielsetzung, Fragestellungen und Methodik}

Bislang fehlen geeignete Instrumente zur empirisch-quantitativen Erforschung von Einstellungen gegenüber Cybermobbing. Diese Forschungslücke bearbeitend interessiert in diesem Beitrag, welche Einstellungen Schülerinnen und Schüler in der Schweiz gegenüber Cybermobbing (operationalisiert anhand der beiden Formen Flaming und Denigration) haben. Das Ziel des vorliegenden Beitrags liegt somit in der Präsentation der Reliabilität und Konstruktvalidität eines eigenentwickelten Instruments zur Erfassung von Einstellungen gegenüber diesen beiden Cybermobbingformen. Konkret werden in einem ersten Schritt die empirischen Qualitätskriterien des entwickelten Instruments überprüft und daran anschliessend werden in einem zweiten Schritt erste Beschreibungen zu den Einstellungen gegenüber Cybermobbing von Schülerinnen und Schülern in der Schweiz vorgenommen.

\section{Stichprobe}

Die Betrachtung der Schülerinnen und Schüler der Schweiz konzentriert sich auf die Sekundarstufe I, wobei zwei verschiedene Stichproben herangezogen werden, welche aus zwei grossen Vergleichsstudien entstammen. Einerseits konnten im Rahmen von ICILS (International Computer and Information Literacy Study) der IEA (International Association for the Evaluation of Educational Achievement) basierend auf einem nationalen Modul Schülerinnen und Schüler der achten Jahrgangsstufe befragt werden. Die Aussagen zu den Einstellungen gegenüber Cybermobbing haben 2915 der insgesamt 3225 in ICILS beteiligten Schülerinnen und Schüler aus 98 Schulen der Schweiz beantwortet (siehe Tab. 1). ${ }^{2}$ Das Durchschnittsalter dieser Schülerinnen und Schüler liegt bei 14.3 Jahren (ICILS insgesamt: 14.7 Jahre) und der Anteil an Mädchen bei 49 Prozent (ICILS: 48 Prozent). In Betrachtung der Sprachregionen fällt auf, dass die Anzahl an Schülerinnen und Schüler aus der italienischsprachigen Schweiz im Vergleich mit den anderen beiden Sprachregionen hoch ausfällt. Der Grund dafür liegt in einer Vollerhebung in dieser Sprachregion. ${ }^{3}$ Die Datenerhebung erfolgte anhand einer Fragebogenerhebung.

Andererseits konnten die hier interessierenden Einstellungen gegenüber Cybermobbing auch im Rahmen der Studie «Lebenswelten Ostschweizer Jugendlicher» (LeOJ) (Beck \& Ha, in Druck) in das Erhebungsinstrument aufgenommen werden. Dabei wurden insgesamt 1333 Jugendliche aus 81 Klassen der Sekundar- und Realschule aus den Kantonen St.Gallen und AppenzellAusserrhoden befragt. ${ }^{4}$ Das Befragungsmodul, welches die Items zu den Einstellungen gegenüber den beiden Cybermobbingformen beinhaltete, wurde insgesamt von 472 Schülerinnen und Schülern bearbeitet. Diese haben ein mittleres Alter von 13.7 Jahren (LeOJ insgesamt: 13.7 Jahre), wobei sich die Spannweite von 12 bis 16 Jahren zeigt (LeOJ insgesamt: 11 bis 16 Jahre). Der 
Anteil an Mädchen und Jungen fällt mit je 50 Prozent sowohl insgesamt wie in der hier interessierenden Stichprobe identisch aus. Die Befragung fand als Querschnittsstudie am Ende des Jahres 2016 statt und wurde als klassenraumbasierte Onlinebefragung durchgeführt (siehe Tab. 1).

Tabelle 1: Merkmale der Schülerinnen und Schüler der Schweizer Stichproben in ICILS 2013 und LeOJ: insgesamt und für die hier vorliegenden Analysen

\begin{tabular}{llll}
\hline & Anzahl & \% Mädchen & $\begin{array}{l}\text { Alter } \\
\text { Mittelwert }\end{array}$ \\
ICILS Stichprobe insgesamt & 3225 & $48 \%$ & 14.69 \\
\hline Deutschsprachige Schweiz & 1159 & $49 \%$ & 14.75 \\
$\quad$ Französischsprachige Schweiz & 971 & $48 \%$ & 14.51 \\
$\quad$ Italienischsprachige Schweiz & 1095 & $46 \%$ & 14.50 \\
\hline $\begin{array}{l}\text { ICILS Nationales Modul: } \\
\text { Einstellung ggn. Cybermobbing }\end{array}$ & $\mathbf{2 9 1 5}$ & $\mathbf{4 9 \%}$ & $\mathbf{1 4 . 2 9}$ \\
\hline $\begin{array}{l}\text { Lebenswelten Ostschweizer Jugendlicher } \\
\text { (LeOJ) insgesamt }\end{array}$ & 1333 & $50 \%$ & 13.73 \\
\hline $\begin{array}{l}\text { LeOJ: Modul } \\
\text { Einstellung ggn. Cybermobbing }\end{array}$ & 472 & $50 \%$ & 13.74 \\
\hline
\end{tabular}

\section{Instrumente}

Die Skala zur Erfassung der Einstellung gegenüber Cybermobbing basiert auf fünf vorgegebenen Aussagen, welche anhand einer standardisierten Likert-Skala mit vierstufigem Antwortformat hinsichtlich der subjektiven Zustimmung bzw. Ablehnung (stimme gar nicht zu bis stimme völlig zu) bewertet wurden (siehe Tab. 2). Die subjektive Erfahrung mit Cybermobbing wurde mit Bezug auf eine vorgelegte Vignette erfragt. Darin wird geschildert, wie Schülerinnen und Schüler auf Facebook einen Mitschüler verhandeln und über ihn lästern, wodurch es auch zu Momenten des Lästerns und des Ausschlusses im «realen» Leben kommt. Die Schülerinnen und Schüler wurden gefragt, ob sie eine derartige oder ähnliche Situation (a) schon beobachten konnten, (b) schon mitgemacht haben und (c) schon selbst erfahren mussten. Aufgrund der damit verbundenen eingeschränkten Erfassung der Erfahrungen mit Cybermobbing hinsichtlich der Formen und Bereiche, kann «nur» von einer Annäherung an die Erfahrungen insgesamt ausgegangen werden.

\section{Datenauswertungen}

Wie bereits erwähnt, besteht das Ziel dieses Beitrags in der Instrumentenvalidierung der eigenentwickelten Skala zur Einstellung gegenüber Cybermobbing - was im Querschnitt, basierend auf zwei unabhängigen Stichproben, vorgenommen wird. Um die Dimensionalität der Skala zu prüfen und - wie postuliert - einen homogenen Faktor zu identifizieren, wurden zunächst explorative 
Faktorenanalysen (EFA) berechnet. Auf der Grundlage dieser Ergebnisse wurden in einem zweiten Schritt konfirmatorische Faktorenanalysen (KFA) für das Konstrukt der Einstellung gegenüber Cybermobbing spezifiziert. Zur Analyse der Gruppenvergleiche wurden einfaktorielle Varianzanalysen (ANOVA) mit der Statistiksoftware SPSS (Version 22) getestet. Die Analysen zur Faktoren-struktur erfolgten mit dem Programm Mplus (Muthén \& Muthén, 2012).

\section{Ergebnisse}

\section{Einstellung gegenüber Cybermobbing: Skalenanalyse und Messmodell}

\section{Explorative Faktorenanalyse}

In inhaltlicher Betrachtung der fünf Einzelitems der Skala Einstellung gegenüber Cybermobbing kann erkannt werden, dass sich diese auf die beiden Formen «Flaming» (Beleidigung, Beschimpfung) und «Denigration» (Verunglimpflichung, Anschwärzen, Gerüchte verbreiten) aus der Klassifikation nach Willard (2007; siehe Abschnitt 2) beziehen (siehe Tab. 2).

Tabelle 2: Skala Einstellung gegenüber Cybermobbing - Items und Antwortkategorien sowie Skalenkennwerte und Reliabilitäten für die beiden Stichproben

Items Antwortkategorien

Es ist in Ordnung im Internet über arrogante Leute zu lästern. $(\mathrm{cm} 1)$

\begin{tabular}{|c|c|}
\hline $\begin{array}{l}\text { Wenn mir jemand etwas zuleide getan hat, darf ich über ihn/sie im } \\
\text { Internet lästern. }(\mathrm{cm} 2)\end{array}$ & \multirow{4}{*}{$\begin{array}{l}1=\text { stimme gar nicht } \mathrm{zu} \\
2=\text { stimme eher nicht } \mathrm{zu} \\
3=\text { stimme eher } \mathrm{zu} \\
4=\text { stimme völlig } \mathrm{zu}\end{array}$} \\
\hline $\begin{array}{l}\text { Ich darf im Internet jemanden blossstellen, wenn ich sonst keine } \\
\text { Möglichkeit habe mit ihm zu streiten. }(\mathrm{cm} 3)\end{array}$ & \\
\hline In Blogs über andere Personen zu lästern, ist nur Spass. (cm4) & \\
\hline $\begin{array}{l}\text { Es ist in Ordnung, jemanden den ich nicht mag im Internet (z.B. } \\
\text { Facebook, Twitter) zu verhöhnen. (cm5) }\end{array}$ & \\
\hline
\end{tabular}

Skala (ICILS) $: N=2915, M=1.81, S D=.76, S E=.01$, Min $=1$, Max $=4, \alpha=.84$ Skala $($ LeOJ) $: N=472, M=1.40, S D=.61, S E=.03$, Min $=1$, Max $=4, \alpha=.87$

Anmerkung: $\mathrm{N}=$ Fallzahl, $\mathrm{M}=$ Mittelwert, $\mathrm{SD}=$ Standardabweichung, $\mathrm{SE}=$ Standardfehler, $\mathrm{Min}=$ Minimum, Max = Maximum, $\alpha=$ Cronbachs Alpha

Zur Untersuchung der Faktorenstruktur dieser Einzelitems wurde in einem ersten Schritt getrennt für die beiden unabhängigen Stichproben eine Hauptkomponentenanalyse (PCA) durchgeführt. Die Items wurden mittels einer schiefwinkligen Promax-Rotation in Richtung eines Einfachladungsmusters der 
manifesten Variablen rotiert. Faktorladungen von grösser .50 wurden hierbei als bedeutsam erachtet (z.B. Backhaus, Erichson, Plinke \& Weiber, 2010; Bortz \& Schuster, 2010; Bühner, 2011). Als statistisches Kriterium für die Anzahl der zu extrahierenden Faktoren wurden Eigenwerte grösser Eins (Kaiser-Guttmann Kriterium) gewählt. Die Analyse extrahierte für beide Stichproben die theoretisch postulierte einfaktorielle Lösung mit Eigenwerten von 3.02 (ICILS: Varianzaufklärung von 60,3 Prozent) und 3.38 (LeOJ: Varianzaufklärung von 67,7 Prozent) sowie zufriedenstellenden Gütekriterien (ICILS: Stichprobeneignung nach Kaiser-Meyer-Olkin $=.85$; Bartlett-Test auf Sphärizität $\chi^{2}{ }_{10}=$ 5,044.85, $p<.001$; LeOJ: Stichprobeneignung nach Kaiser-Meyer-Olkin = .86; Bartlett-Test auf Sphärizität $\left.\chi^{2}{ }_{10}=1209.46, \mathrm{p}<.001\right)$. Eine explorative Faktorenanalyse (EFA) zeigt des Weiteren, dass alle Items (im Folgenden jeweils mit $\mathrm{cm} 1$ bis $\mathrm{cm} 5$ bezeichnet) auf einem Faktor mit Faktorladungen von jeweils über .76 laden $\left(\right.$ ICILS: $\mathrm{cm} 1_{\text {ICILS }}=.78, h^{2}=.60 ; \mathrm{cm} 2_{\text {ICILS }}=.79, h^{2}=.62 ; \mathrm{cm} 3_{\text {ICILS }}=$ $.78, h^{2}=.60 ; \mathrm{cm} 4_{\mathrm{ICILS}}=.78, h^{2}=.60 ; \mathrm{cm} 5_{\mathrm{ICILS}}=.76, h^{2}=.58 ; \mathrm{LeOJ}: \mathrm{cm}^{\mathrm{LeOJ}}=$ $.80, h^{2}=.64 ; \mathrm{cm} 2_{\mathrm{LeOJ}}=.85, h^{2}=.72 ; \mathrm{cm} 3_{\mathrm{LeOJ}}=.83, h^{2}=.68 ; \mathrm{cm} 4_{\mathrm{LeOJ}}=.82, h^{2}$ $\left.=.67 ; \mathrm{cm} 5_{\mathrm{LeOJ}}=.82, h^{2}=.67\right)$. Die Reliabilitätsanalyse bestätigt ferner, dass mit Hilfe der fünf Items die Einstellung gegenüber Cybermobbing gut abgebildet werden kann, wobei die interne Konsistenz der Skala bzw. die Skalengüte sowohl für ICILS $(\alpha=.84)$ als auch für LeOJ $(\alpha=.87)$ als hoch einzustufen ist.

\section{Konfirmatorische Faktorenanalyse}

Die theoretisch postulierte Eindimensionalität des Konstruktes zur Einstellung gegenüber Cybermobbing wurde anschliessend mit Hilfe einer konfirmatorischen Faktorenanalyse (KFA) mittels Maximum-Likelihood-Methode geprüft (z.B. Bollen, 1989; Byrne, 2003, 2011; Geiser, 2011; Kline, 2011; Reinecke \& Pöge, 2010). Fehlende Werte wurden dabei mittels des Full Information Maximum Likelihood (FIML-)Verfahrens geschätzt (Lüdtke \& Robitzsch, 2010a, 2010b; Lüdtke, Robitzsch, Trautwein \& Köller, 2007). Für beide Stichproben bestätigte sich die Eindimensionalität des Konstrukts. Das in Form eines g-Faktor-Modells (Generalfaktorenmodell) dargestellte Modell weist für beide Stichproben hohe Faktorladungen für die fünf Indikatoren auf (ICILS: $\lambda=.69$ bis $\lambda=.73$ [siehe Abb. 1]; LeOJ: $\lambda_{\mathrm{cm} 1}=.72$, Residuum ${ }_{\mathrm{cm} 1}=.49 ; \lambda_{\mathrm{cm} 2}=.72$, Residuum $_{\mathrm{cm} 2}=.48 ; \lambda_{\mathrm{cm} 3}=.79$, Residuum ${ }_{\mathrm{cm} 3}=.37 ; \lambda_{\mathrm{cm} 4}=.84$, Residuum $_{\mathrm{cm} 4}=$ $.30 ; \lambda_{\mathrm{cm} 5}=.75$, Residuum $\left._{\mathrm{cm} 5}=.43\right)$. Die Standardfehler variieren zwischen .013 und .018 (ICILS) bzw. zwischen .072 und .078 (LeOJ). 


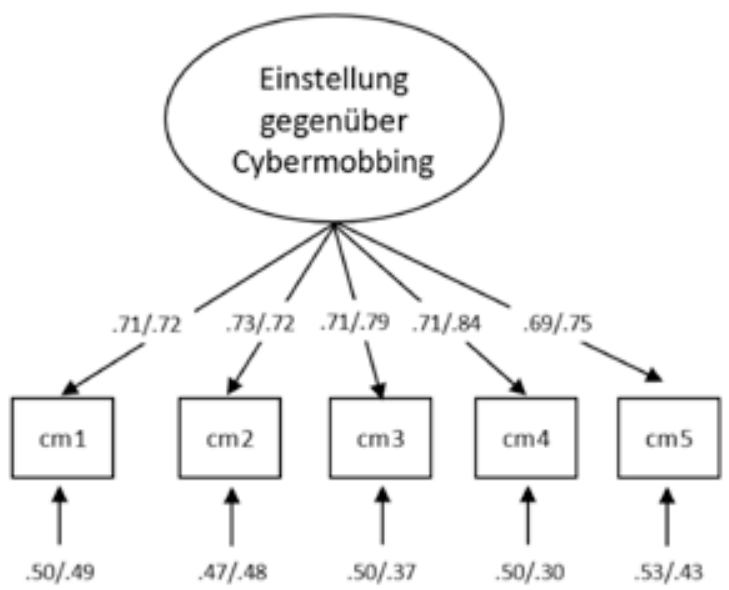

Abbildung 1: Generalfaktor-Modell der Einstellung gegenüber Cybermobbing (ICILS/LeOJ)

In beiden Datengrundlagen tragen alle Indikatoren signifikant $(p<.001)$ zur Varianzaufklärung der Einstellung gegenüber Cybermobbing bei, wie aus Tabelle 3 entnommen werden kann, in welcher die standardisierten Intercepts, Residualvarianzen und $\mathrm{R}^{2}$-Werte des Generalfaktorenmodells aufgeführt werden.

Tabelle 3: Intercepts, Residualvarianzen und $R^{2}$ der Items des Konstrukts der Einstellung gegenüber Cybermobbing in den Studien ICILS und LeOJ

\begin{tabular}{llll}
\hline & Intercept $(\mathrm{SE})$ & Residualvarianz $(\mathrm{SE})$ & $\mathrm{R}^{2}(\mathrm{SE})$ \\
\hline $\mathrm{cm} 1$ (ICILS) & $1.74^{* * *}(.013)$ & $.50^{* * *}(.021)$ & $.51^{* * *}(.021)$ \\
$\mathrm{cm} 1$ (LeOJ) & $1.92^{* * *}(.076)$ & $.49^{* * *}(.038)$ & $.51^{* * *}(.038)$ \\
\hline $\mathrm{cm}$ 2 (ICILS) & $1.95^{* * *}(.018)$ & $.47^{* * *}(.022)$ & $.53^{* * *}(.022)$ \\
$\mathrm{cm}$ 2 (LeOJ) & $2.00^{* * *}(.078)$ & $.48^{* * *}(.038)$ & $.52^{* * *}(.038)$ \\
\hline $\mathrm{cm}$ 3 (ICILS) & $1.83^{* * *}(.015)$ & $.50^{* * *}(.022)$ & $.51^{* * *}(.022)$ \\
$\mathrm{cm}$ 3 (LeOJ) & $1.97^{* * *}(.077)$ & $.37^{* * *}(.034)$ & $.63^{* * *}(.034)$ \\
\hline $\mathrm{cm} 4$ (ICILS) & $1.92^{* * *}(.017)$ & $.50^{* * *}(.020)$ & $.50^{* * *}(.020)$ \\
$\mathrm{cm} 4$ (LeOJ) & $1.90^{* * *}(.075)$ & $.30^{* * *}(.032)$ & $.70^{* * *}(.032)$ \\
\hline $\mathrm{cm} 5$ (ICILS) & $1.88^{* * *}(.016)$ & $.53^{* * *}(.021)$ & $.48^{* * *}(.021)$ \\
$\mathrm{cm} 5$ (LeOJ) & $1.75^{* * *}(.072)$ & $.43^{* * *}(.036)$ & $.57^{* * *}(.036)$ \\
\hline
\end{tabular}

Anmerkung: ${ }^{* *} \mathrm{~cm} 1$ bis $\mathrm{cm} 5=$ Item 1 bis Item $5, \mathrm{p}<.001, \mathrm{SE}=$ Standardfehler, $\mathrm{R}^{2}=$ Varianzaufklärung

Für die Quantifizierung der Datenanpassung wurde die Modellanpassungsgüte über die Modellfit-Indizes $\chi^{2}$-Test, CFI, RMSEA und SRMR überprüft. ${ }^{5} \mathrm{Als}$ Cutoff-Kriterien für die Modellbeurteilung wurden die Empfehlungen von $\mathrm{Hu}$ und Bentler (1999) herangezogen. ${ }^{6}$ Die Fit-Indizes verweisen insgesamt auf eine 
gute Modellanpassungsgüte an die Daten. Das strukturüberprüfende Verfahren der konfirmatorischen Faktorenanalyse bestätigt in beiden Datensätzen das theoretisch begründete g-Faktormodell (Modell-Fit - ICILS: $\chi^{2}=9.02 ; d f=5$; $p=.000 ; \mathrm{CFI}=.981 ; \mathrm{RMSEA}=.062 ; \mathrm{SRMR}=.020 ; \mathrm{LeOJ}: \chi^{2}=7.83 ; d f=4$; $p=.10 ; \mathrm{CFI}=.99 ; \mathrm{RMSEA}=.04 ; \mathrm{SRMR}=.01$ ).

\section{Einstellung gegenüber Cybermobbing - Deskriptive Ergebnisse und Gruppenvergleiche}

In ICILS wird die Einstellung gegenüber Cybermobbing von den befragten Schülerinnen und Schülern mit einem Mittelwert von $1.81(S D=.76 ; S E=.01)$ (eher) ablehnend bewertet. Ähnlich zeigt sich auch das Ergebnis für die Studie LeOJ, wobei sich hier mit einem Mittelwert von $M=1.40(S D=.61 ; S E=.03)$ sogar noch ein etwas stärker ablehnendes Ergebnis offenbart (siehe Tab. 2).

In Betrachtung der einzelnen Items, die das Konstrukt der Einstellung gegenüber Cybermobbing abbilden, zeigt sich, dass diesen ca. die Hälfte der Schülerinnen und Schüler jeweils gar nicht und ca. ein Viertel eher nicht zustimmt (siehe Abb. 2). Somit kann festgehalten werden, dass einerseits - wie oben erwähnt - zwar eine deutliche Mehrheit der Schülerinnen und Schüler eine ablehnende Einstellung gegenüber Cybermobbing zum Ausdruck bringt, dass andererseits aber dennoch jeweils ca. ein Viertel der Schülerinnen und Schüler den Aussagen zustimmt und somit gegenüber einem Verhalten der Beleidigung und des Beschimpfens sowie der Verunglimpfung, des Anschwärzens und des Gerüchteverbreitens im Internet nicht negativ eingestellt ist.

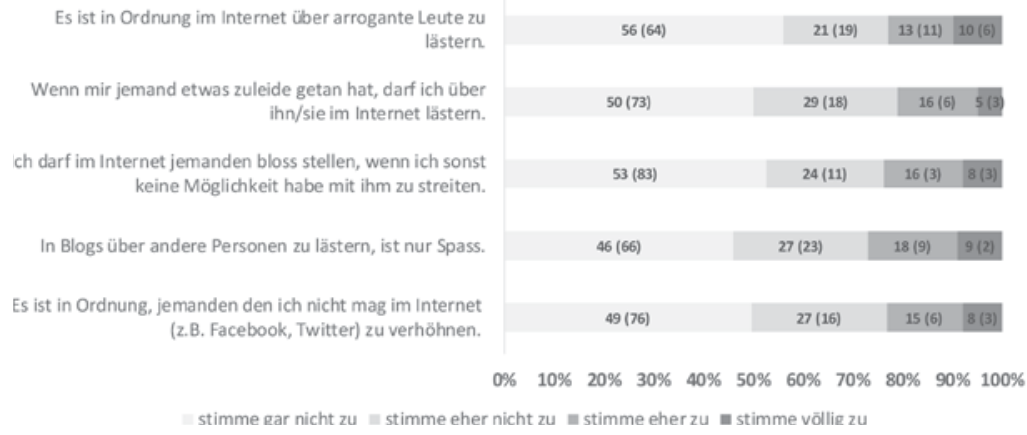

Anmerkung: Werte ohne Klammer bilden die Häufigkeiten aus ICILS ab, jene mit Klammer die Häufigkeiten aus LeOJ

\section{Abbildung 2: Einstellungen gegenüber Cybermobbing - Häufgkeitsverteilung der Einzelitems aus ICILS und LeOJ}

Ein Vergleich der Geschlechter lässt erkennen, dass zwar auch Jungen im Durchschnitt eine ablehnende Einstellung gegenüber Cybermobbing zum Ausdruck bringen, diese aber nicht so eindeutig - also weniger kritisch - ausfällt wie/als 
bei den Mädchen (ICILS: $t(2896)=-9.55, p<.001 ; M_{\text {Mädchen }}=1.67, S D=.69$; $M_{\text {Jungen }}=1.94, S D=.79 \mid d=.36[$ Cohens $d$ ] 7 ; LeOJ: $t(472)=5.02, p<.001$; $\left.M_{\text {Mädchen }}=1.26, S D=.50 ; M_{\text {Jungen }}=1.52, S D=.62 \mid d=.46\right)$.

Die Einstellungen gegenüber Cybermobbingformen nach dem subjektiven Erfahrungshintergrund betrachtet, erhärtet sich das oben dargestellte Ergebnis. Zwar liegen die Mittelwerte für alle Gruppen (Täterinnen und Täter, Opfer, Bystander und Unbeteiligte) noch immer im (eher) ablehnenden Bereich, dies aber in unterschiedlicher Deutlichkeit (siehe Tab. 4). Die geringste Ablehnung findet sich für die Schülerinnen und Schüler, welche nur Erfahrungen als Täterinnen bzw. Täter besitzen. Mit einem Mittelwert von $2.19(S D=.81)$ liegen sie im Zwischenraum von «stimme eher nicht zu» (Wert 2) und «stimme eher zu» (Wert 3), wobei auch hier die Nähe zu «stimme eher nicht zu» noch gegeben ist. Dennoch sprechen wir hier von einer negativen Einstellung, da Cybermobbing als Möglichkeit der Auseinandersetzung nicht eindeutig abgelehnt wird (als diesbezüglich normativ gesetzter Grenzwert bezogen auf die vorliegende Skala gilt grösser zwei). Mit einem ähnlichen Mittelwert von $2.09(S D=.84)$ zeigt sich die Gruppe der Schülerinnen und Schüler, welche sowohl Erfahrungen als Täterinnen und Täter als auch als Opfer besitzen. Demgegenüber fallen die Ablehnungen von Cybermobbing bei den Gruppen "Unbeteiligte» $(M=1.71$; $S D=.70)$, «nur Beobachtende/Bystander» $(M=1.69 ; S D=.68)$ sowie «nur Opfer» $(M=1.67 ; S D=.66)$ noch wesentlich deutlicher aus. Es schälen sich zwei zu unterscheidende Gruppierungen heraus: zum einen die Täterinnen bzw. Täter, einschliesslich derjenigen, die bereits unter Cybermobbing gelitten haben, und zum anderen die Unbeteiligten, Beobachtenden bzw. Bystander und Opfer. Dabei fallen die Unterschiede zwischen den Gruppen zwar statistisch signifikant, jedoch in nur geringer Effektstärke aus $\left(F[4,2375]=18.72 ; p<.001 ; \eta^{2}=.03\right) .{ }^{8}$

Tabelle 4: Varianzanalytische Betrachtung von Personengruppen mit unterschiedlichen Cybermobbing-Erfahrungen bezüglich deren Einstellungen gegenüber Cybermobbing (ICILS-Stichprobe)

\begin{tabular}{l|l|l|l|l}
\hline & $\mathbf{N}$ & $\boldsymbol{M}$ & $\boldsymbol{S D}$ & $\boldsymbol{p}<.05$ \\
\hline Bystander (A) & 434 & 1.69 & .68 & A - B \& A - D \\
\hline Nur Täter/in (B) & 130 & 2.19 & .81 & $\begin{array}{l}\text { B - A \& B - C \& } \\
\text { B - E }\end{array}$ \\
\hline Nur Opfer (C) & 213 & 1.67 & .66 & C - B \& C - D \\
\hline Täter/in-Opfer (D) & 52 & 2.09 & .84 & $\begin{array}{l}\text { D - A \& D - C } \\
\text { \& D - E }\end{array}$ \\
\hline Unbeteiligte (E) & 1551 & 1.71 & .70 & E - B \& E - D \\
\hline
\end{tabular}

Anmerkung: ANOVA - einfaktorielle Varianzanalyse mit Post-Hoc-Tests (Scheffé-Prozedur) ${ }^{1} \mid \mathrm{N}=$ Fallzahl, M = Mittelwert, $S D$ = Standardabweichung, $\mathrm{p}=$ Irrtumswahrscheinlichkeit

${ }^{1}$ Dieser Post-Hoc-Test lässt sich im Rahmen einer Varianzanalyse zur Bestimmung von signifikanten Unterschieden zwischen Gruppenmittelwerten einsetzen. Der Scheffé-Test wird zu den konservativsten Post-Hoc-Tests gezählt. 


\section{Zusammenfassung und Diskussion}

Das Aufwachsen im heutigen «Internetzeitalter» (Buermann, 2012, S. 246) ist wie einleitend gesagt - stark durch neue Medien geprägt, so dass Smartphones, Tablets und generell Internetzugänge zu ständigen (Weg-)Begleitern des Alltags von jungen Menschen geworden sind (Feierabend, Plankenhorn \& Rathgeb, 2016; Organisation for Economic Co-operation and Development [OECD], 2015). Basierend auf den Möglichkeiten dieser technologischen Revolution und einer ständigen Mediatisierung des Alltags finden soziale Interaktionen heutzutage meist über Chatportale statt, welche jedoch auch dysfunktionale Ausmasse von Ausgrenzung, Gewalt und Mobbing mit sich bringen können (z.B. Berne et al., 2013; Katzer, 2014; Katzer, Fetchenhauer \& Belschak, 2009; Olweus, 2012; Pieschl, Porsch, Kahl \& Klockenbusch, 2013; Sticca et al., 2013). Besonderheiten des Cybermobbings entstehen aus der räumlichen und zeitlichen Entgrenzung durch die Kommunikationsmedien. Wie oben aufgezeigt können dabei verschiedene Formen von Cybermobbing unterschieden werden (Willard, 2007) - wobei sich die Operationalisierungen in diesem Beitrag insbesondere auf die Einstellung gegenüber den Formen der Beleidigung und des Beschimpfens («Flaming») sowie der Verunglimpfung, des Anschwärzens und des Gerüchteverbreitens («Denigration») in der Virtualität beziehen. Diese Problematik aufnehmend interessierte in diesem Beitrag, (a) ob es gelingen kann, Einstellungen gegenüber Cybermobbing empirisch zu erfassen und (b) zu ergründen, wie verbreitet Einstellungen gegenüber Cybermobbing bei Schülerinnen und Schülern der Sekundarstufe I der Schweiz sind (betrachtet anhand von zwei anfallenden Stichproben). Die analytischen Betrachtungen differenzieren hierbei zwischen (a) Beobachtenden (sog. Bystander), (b) Opfern, (c) Täterinnen und Tätern sowie (d) Schülerinnen und Schülern die sowohl Opfer als auch Täterinnen und Täter von Cybermobbing waren. Wird Cybermobbing als Möglichkeit der Auseinandersetzung bejaht, sprechen wir von einer negativen Einstellung - in normativer Setzung betrachten wir den diesbezüglichen Grenzwert auf unsere Skala bezogen bei grösser zwei (und damit mit Tendenz zu «stimme der Befürwortung einer derartigen Möglichkeit einer Auseinandersetzung eher zu»).

Die Ergebnisse zeigen, dass Einstellung gegenüber Cybermobbing (mit Blick auf "Flaming» und "Denigration») anhand von fünf Items erhoben werden kann. Das strukturüberprüfende Verfahren der konfirmatorischen Faktorenanalyse bestätigt das theoretisch begründete $g$-Faktormodell mit zufriedenstellenden Fit-Indizes für beide unabhängigen Stichproben.

Insgesamt äussern die befragten Schülerinnen und Schüler (eher) ablehnende Einstellungen gegenüber Cybermobbing. Dabei erweisen sich die Mädchen als noch abweisender als die Jungen. In Differenzierung der Schülerinnen und Schüler nach deren Erfahrungen mit Cybermobbing (Täterschaft, Opfer, Bystander) zeigt sich, dass bei vorliegender Täterschaft die Einstellung weniger ablehnend ausfällt. 
Auch wenn sich insgesamt eine deutliche Mehrheit an Schülerinnen und Schüler zeigt, die gegenüber Cybermobbing bzw. den hier betrachteten Formen «Flaming» und «Denigration ablehnend eingestellt sind, so ist es dennoch ein knappes Viertel, die ein derartiges Verhalten (eher) nicht als verwerflich bewertet. Angesichts der Leiden der Opfer, die immer wieder mahnend (und gelegentlich auch anprangernd) ausgewiesen und dokumentiert werden, so dass diese auch dem grossen Teil der Schülerinnen und Schülern bekannt sein sollten, vermag die doch grosse Zahl an wenig kritischen Stimmen zu erstaunen. Inwieweit derartige Einstellungen darauf gründen, dass diese jungen Menschen Defizite in den Bereichen der (sozialen) Perspektivenübernahme (Selman, 1980, 2003) und der Empathiefähigkeit besitzen, und/oder sich primär in virtuellen Welten bewegen und dadurch Bezüge zur realen Welt deutlich abgeschwächt vorhanden sind, muss offenbleiben und kann anhand der bestehenden Daten nicht überprüft werden. Deutlich machen die Ergebnisse jedoch, dass sich Erziehungs- und Bildungsverantwortliche weiterhin aktiv um einen verantwortungsvollen Umgang mit neuen Technologien bemühen müssen. Um den negativen Auswirkungen von Cybermobbing frühzeitig gerecht werden zu können, bedarf es dabei auch evidenzbasierter Präventions- und Interventionsprogramme (z.B. Gradinger, Yanagida \& Strohmeier, 2014; Hinduja \& Patchin, 2009; Pfetsch, Mohr \& Ittel, 2012, 2014; Pieschl \& Porsch, 2012). Diese sollten bereits im jungen Kindesalter starten, in welchem (noch) weniger allfälliges Fehlverhalten in den Blick genommen werden muss, sondern gezielt an den sich entwickelnden handlungsleitenden Einstellungen (Ajzen, 1985, 1991) gearbeitet werden kann.

Trotz oder gerade aufgrund der präsentierten statistisch bedeutsamen Ergebnisse gilt es abschliessend nochmals darauf hinzuweisen, dass diese nicht repräsentativ für die Schweiz betrachtet werden können. Auch muss berücksichtigt werden, dass die Erfassung der Einstellungen gegenüber Cybermobbing auf fünf Items basiert, die (nur) die Formen der Beleidigung und des Beschimpfens sowie der Verunglimpfung, des Anschwärzens und des Gerüchteverbreitens beinhalten - was nach Willard (2007) lediglich zwei der sieben Formen entsprechen kann. All diese aufgeführten Punkte stellen weitere Forschungsdesiderata dar, welche in nächsten Schritten differenziert bearbeitet werden sollen.

Aufgrund der erwähnten Limitationen können aus den vorliegenden Befunden keine direkten Empfehlungen abgeleitet werden. Desiderata können jedoch formuliert werden. Diese müssen aber dergestalt sein, dass sie auch hier nicht besprochene Befunde und Erfahrungen zusätzlich aufgreifen:

1. Es ist bedeutungsvoll, dass Lehrpersonen über das Phänomen des Cybermobbings gut informiert sind, weshalb sie in der Lehrerinnen- und Lehrerbildung sowie -weiterbildung zur Sprache kommen müssen. Dadurch soll garantiert werden, dass sie verstehen, was im Umgang mit neuen Technologien passieren kann bzw. passiert. 
2. Es ist bedeutungsvoll, dass Lehrpersonen diagnostische Verfahren kennenlernen, die auf Ausschluss (und möglichst auch auf Täterschaft) von Kindern und Jugendlichen durch Cybermobbing hinweisen.

3. Es ist wünschenswert, dass Lehrpersonen verschiedene interventive Verfahren zum Stoppen oder präventiv zum Verhindern solcher Prozesse kennenlernen. So ist beispielsweise der No-Blame-Approach (Blum \& Beck, 2012), der ohne Schuldzuweisung arbeitet, nur eine von vielen Möglichkeiten, sinnvoll zu reagieren.

4. Es wäre wünschenswert, ein Forschungsschwerpunktprogramm zum Thema Cybermobbing zu lancieren. Wir wissen noch viel zu wenig über die Bedingungen der Entstehung des Phänomens, dies sowohl in Perspektive der in diesem Beitrag primär interessierenden Einstellungen als auch hinsichtlich des konkreten Verhaltens.

\section{Anmerkungen}

1 Beispiele diesbezüglicher Berichterstattungen finden sich auch immer wieder in der medialen Presse, so etwa jenes von Megan Meier, welches international grosse Betroffenheit ausgelöst hat: «Gerade einmal 13 Jahre war Megan Meier alt, als sie sich das Leben nahm. Dabei hing der Himmel für sie bis kurz vor dem Selbstmord noch voller Geigen. Grund dafür war Josh, eine Internet-Bekanntschaft aus MySpace. Josh und Megan sendeten sich Nachrichten, das Mädchen war über beide Ohren verliebt - und mit ihren 13 Jahren eigentlich noch gar nicht dazu berechtigt, einen MySpace-Account zu unterhalten. Das Problem war nur, Josh existierte gar nicht! Eine ehemalige Freundin von Megan hatte sich den charmanten Jungen ausgedacht, der sich für die übergewichtige, depressive Megan interessierte. Und plötzlich änderte sich der Tonfall: Jetzt wurde Megan als fett und Schlampe beschimpft. Für die sensible Schülerin einer katholischen Privatschule war das zu viel, sie wollte nicht mehr leben. Besonders perfid: Die Mutter des hinter dem Streich steckenden Mädchens half bei der Demütigung mit. Sie wollte auf diese Weise herausfinden, was Megan denn so hinter dem Rücken über ihre Tochter sage. Mit fatalen Folgen. - Das Drama geschah am 16. Oktober letzten Jahres, als ihre Eltern Megan erhängt in ihrem Zimmer fanden (...)» (Blick, 03.01.2012). Cybermobbing als Form diskriminierender und belästigender Demütigung und Diffamierung über elektronische Medien wie Chatrooms und Messaging ist zu einem gesellschaftlichen Täter-Opfer-Syndrom geworden.

2 In der Schweiz kann die ICILS-Stichprobe aufgrund von Problemen in der Gewinnung der zufällig gezogenen Schulen «nur» als anfallend und nicht als repräsentativ interpretiert werden. Dadurch können auch die Ergebnisse aus ICILS nicht als national repräsentativ, sondern vielmehr als (starke) Tendenzen interpretiert werden.

3 Diese Überrepräsentation der italienischsprachigen Schweiz müsste unter dem Gesichtspunkt einer nationalen Repräsentativität der Ergebnisse «korrigiert» werden (anhand von Gewichtungen der einzelnen Schülerinnen und Schüler). Da es sich jedoch «nur» um eine anfallende Stichprobe mit tendenziellen und nicht generalisierbaren Ergebnissen handelt, wird darauf verzichtet.

4 Im Kanton St.Gallen nahmen 65 Schulklassen und im Kanton Appenzell-Ausserrhoden 16 Schulklassen an der Jugendstudie teil.

5 Beauducel und Wittmann (2005) empfehlen den $\chi^{2}$-Wert und den p-Wert, RMSEA (Root Mean Square Error of Approximation), CFI (Comparative Fit Index) sowie SRMR 
(Standardized Root Mean Residual) als Fit-Indizes zu berichten. Nach Bühner (2011) liefern diese Fit-Indizes verschiedene Informationen über die Modellpassung.

6 Nach Hu und Bentler (1999) können für die Bewertung der Modellanpassungen folgende Kriterien zu Grunde gelegt werden: CFI $\geq .96$, RMSEA $\leq .05$, SRMR $\leq .08$.

7 Cohen (1988) unterscheidet die folgenden Effektstärken: kleiner Effekt: $d=.20$; mittlerer Effekt: $d=.50$; starker Effekt: $d=.80$.

8 Die Erfahrung mit Cybermobbing wurde nur im Rahmen von ICILS erfasst, weshalb sich die darauf bezogenen Ergebnisse auf diese Studie reduzieren.

\section{Literatur}

Ajzen, I. (1985). From intentions to action: a theory of planned behavior. In J. Huhl, \& J. Beckman (Eds.), Will; performance; control (psychology); motivation (psychology) (pp. 11-39). Berlin: Springer Verlag.

Ajzen, I. (1991). The theory of planned behavior. Organizational Behavior and Human Decision Processes, 50, (2), 179-211.

Backhaus, K., Erichson, B., Plinke, W. \& Weiber, R. (2010). Multivariate Analysemethoden. Eine anwendungsorientierte Einführung (13., überarbeitete Auflage). Berlin: Springer.

Baier, D., Krenz, M. \& Bergmann, M. C. (2016). Verbreitung und Einflussfaktoren des Cyberbullyings: Ergebnisse einer Repräsentativbefragung in Niedersachsen. Zeitschrift für Soziologie der Erziehung und Sozialisation, 36, (3), 227-245.

Beauducel, A. \& Wittmann, W. W. (2005). Simulation study on fit indices in confirmatory factor analysis based on data with slightly distorted simple structure. Structural Equation Modeling, 12, 41-75.

Beck, M. \& Ha, J. (in Druck). Lebenswelten Ostschweizer Jugendlicher. St.Gallen: Pädagogische Hochschule St.Gallen.

Bergmann, M. C. \& Baier, D. (2016). Erfahrungen von Jugendlichen mit Cybergrooming: Schülerbefragung - Jugenddelinquenz. Rechtspsychologie, 2, (2), 172-189.

Berne, S., Frisén, A., Schultze-Krumbholz, A., Scheithauer, H., Naruskov, K., Luik, P., Katzer, C., Erentaite, R. \& Zukauskiene, R. (2013). Cyberbullying assessment instruments: A systematic review. Aggression and Violent Behavior, 18, 320-334.

Blick. (2012). Schweizer Tageszeitung Blick vom 3. Januar 2012.

Blum, H. \& Beck, D. (2012). No blame appproach. Mobbing-Intervention in der Schule. Praxishandbuch. Köln: fairaend.

Bollen, K. A. (1989). Structural equations with latent variables. New York, NY: Wiley.

Bortz, J. \& Schuster, C. (2010). Statistik für Human- und Sozialwissenschaftler. Berlin: Springer.

Bühner, M. (2011). Einführung in die Test-und Fragebogenkonstruktion (3., aktualisierte und erweiterte Auflage). München: Pearson Education.

Buermann, U. (2012). Erziehung zur Medienkompetenz. In C. Möller (Hrsg.), Internet- und Computersucht bei Jugendlichen und Heranwachsenden. Ein Praxishandbuch für Therapeuten, Pädagogen und Eltern (S. 242-252). Stuttgart: Kohlhammer.

Byrne, B. M. (2003). Confirmatory factor analysis. In R. Fernandez-Ballesteros (Ed.), Encyclopedia of psychological assessment (Vol. 1, pp. 399-402). Thousand Oaks, CA: Sage.

Byrne, B. M. (2011). Structural equation modeling with Mplus: Basic concepts, applications, and programming. New York, NY: Taylor and Francis.

Cohen, N. J. (1988). Statistical power analysis for the behavioral sciences (2. Aufl.). Hillsdale, NJ: Earlbaum.

DIE ZEIT (2013). Vernetzt und verletzt. ZEIT-ONLINE, 47. Verfügbar unter www.zeit. de/2013/47/phaenomen-cybermobbing

Eagly, A. H. \& Chaiken, S. (1993). The psychology of attitudes. Orlando, FL: Harcourt Brace Jovanovich College. 
Erdur-Baker, Ö. (2010). Cyberbullying and its correlation to traditional bullying, gender and frequent and risky usage of internet-mediated communication tools. New Media \& Society, 12, (1), 109-125.

Erentaité, R., Bergman, L. R. \& Žukauskiené, R. (2012). Cross-contextual stability of bullying victimization: A person-oriented analysis of cyber and traditional bullying experiences among adolescents. Scandinavian Journal of Psychology, 53, 181-190.

Fazio, R. H. \& Petty, R. E. (Eds.). (2007). Attitudes: Their structure, function, and consequences. Hove: Psychology Press.

Feierabend, S., Plankenhorn, T. \& Rathgeb, T. (2016). JIM-Studie 2016 - Jugend, Information, (Multi-) Media. Basisstudie zum Medienumgang 12-bis 19-Jähriger in Deutschland. Stuttgart: Medienpädagogischer Forschungsverbund Südwest.

Fenaughty, J. \& Harré, N. (2013). Factors associated with distressing electronic harassment and cyberbullying. Computers in Human Behavior 29, (1), 803-811.

Festl, R. (2015). Täter im Internet. Eine Analyse individueller und struktureller Erklärungsfaktoren von Cybermobbing im Schulkontext. Wiesbaden: Springer VS.

Festl, R. (2016). Perpetrators on the internet: Analyzing individual and structural explanation factors of cyberbullying in school context. Computers in Human Behavior, 59, 237-248.

Festl, R., Scharkow, M. \& Quandt, T. (2013). Peer influence, internet use and cyberbullying: A comparison of different context effects among German adolescents. Journal of Children and Media, 7, (4), 446-462.

Festl, R., Scharkow, M. \& Quandt, T. (2015). The individual or the group: A multilevel analysis of cyberbullying in school classes. Human Communication Research, 41, (4), 535-556.

Festl, R., Vogelgesang, J., Scharkow, M. \& Quandt, T. (2017). Longitudinal patterns of involvement in cyberbullying: Results from a latent transition analysis. Computers in Human Behavior, 66, 7-15.

Geiser, C. (2011). Datenanalyse mit Mplus: Eine anwendungsorientierte Einführung. Wiesbaden: VS Verlag für Sozialwissenschaften.

Gradinger, P., Strohmeier, D. \& Spiel, C. (2009). Traditional bullying and cyberbullying: Identification of risk groups for adjustment problems. Journal of Psychology, 217, (4), 205-213.

Gradinger, P., Yanagida, T. \& Strohmeier, D. (2014). Evidenzbasierte Prävention und Intervention. Grundlagen und Anwendung am Beispiel Cybermobbing. In T. Porsch \& S. Pieschl (Hrsg.), Neue Medien und deren Schatten: Mediennutzung, Medienwirkung und Medienkompetenz (S. 301-326). Göttingen: Hogrefe.

Gradinger, P., Yanagida, T., Strohmeier, D. \& Spiel, C. (2016). Effectiveness and sustainability of the ViSC Social Competence program to prevent cyberbullying and cyber-victimization: Class and individual level moderators. Aggressive Behavior, 42, 181-193.

Gutzwiller, E. (2015). Moral disengagement and aggression. Comments on the Special Issue. Merrill Palmer Quarterly, 61, (1), 192-211.

Haddock, G. \& Maio, G. R. (2014). Einstellungen. In K. Jonas, W. Stroebe \& M. Hewstone (Hrsg.), Sozialpsychologie (S. 197-229). Heidelberg: Springer.

Hinduja, S. \& Patchin, J. W. (2009): Bullying beyond the schoolyard: Preventing and responding to cyberbullying. Thousand Oaks, CA: Sage Publications.

Hu, L. T. \& Bentler, P. M. (1999). Cutoff criteria for fit indexes in covariance structure analysis: Conventional criteria versus new alternatives. Structural Equation Modeling, 6, (1), 1-55.

Katzer, C. (2014). Cybermobbing - Wenn das Internet zur W@ffe wird. Berlin: Springer Spektrum.

Katzer, C., Fetchenhauer, D. \& Belschak, F. (2009). Cyberbullying. Who are the victims? Journal of Media Psychology, 21, (1), 25-36. 
Kern, S. (2014). Cyber-Mobbing: Mobbing unter Digital Natives - Formen und Folgen von Sozialer Aggression im Internet. Hamburg: Diplomica Verlag.

Kindler, H. (2011). Cyberbullying und Cyberaggression durch Kinder und Jugendliche. In G. Deegener \& W. Körner (Hrsg.), Gewalt und Aggression im Kindes- und Jugendalter. Ursachen, Formen, Intervention (S. 267-283). Weinheim: Beltz.

Kline, R. B. (2011). Principles and practice of structural equation modeling (3. Auflage). New York, NY: Guilford Press.

Kowalski, R. M., Morgan, C. A. \& Limber, S. P. (2012). Traditional bullying as a potential warning sign of cyberbullying. School Psychology International, 33, (5), 505-519.

Langos, C. (2012). Cyberbullying. The challenge to define. Cyberpsychology, Behavior and Social Networking, 15, (6), 285-289.

Leymann, H. (1993). Mobbing. Psychoterror am Arbeitsplatz und wie man sich dagegen wehren kann. Hamburg: Rowohlt.

Lüdtke, O. \& Robitzsch, A. (2010a). Umgang mit fehlenden Daten in der empirischen Bildungsforschung. In Maschke, S. \& Stecher, L. (Hrsg.), Enzyklopädie Erziehungswissenschaft Online. Fachgebiet Methoden der empirischen erziehungswissenschaftlichen Forschung, Quantitative Forschungsmethoden. Weinheim: Juventa.

Lüdtke, O. \& Robitzsch, A. (2010b). Missing-Data-Analyse. In H. Holling \& B. Schmitz (Hrsg.), Handbuch Statistik, Methoden und Evaluation (S. 723-729). Göttingen: Hogrefe.

Lüdtke, O., Robitzsch, A., Trautwein, U. \& Köller, O. (2007). Umgang mit fehlenden Werten in der psychologischen Forschung: Probleme und Lösungen. Psychologische Rundschau, 58, (2), 103-117.

Maio, G. R. \& Haddock, G. (2010). The psychology of attitudes and attitude change. London: Sage.

McQuade, S. C., Colt, J. P. \& Meyer, N. B. (2009). Cyber bullying. Protecting kids and adults from online bullies. Westport, CT: Praeger.

Muthén, L. K. \& Muthén, B. O. (2012). Mplus user's guide (7th ed.). Los Angeles, CA: Muthén \& Muthén.

Neuber, V., Künsting, J. \& Phieler, D. (2014). Cyberbullying unter Schülerinnen und Schülern. Zusammenhänge mit traditionellem Bullying und Persönlichkeitseigenschaften. Psychologie in Erziehung und Unterricht, 2, 81-95.

OECD. (2015). Students, computers and learning: Making the connection. PISA, OECD Publishing. doi:10.1787/9789264239555-en

Olweus, D. (2012). Cyberbullying: An overrated phenomenon? European Journal Of Developmental Psychology, 9, (5), 520-538.

Özsöz, F. (2013). Cyberaggression unter Kindern und Jugendlichen in Deutschland. Recht der Jugend und des Bildungswesens, 61, (2), 182-197.

Perren, S., Corcoran, L., Cowie, H., Dehue, F., Garcia, D., Mc Guckin, C., Sevcikova, A., Tsatsou, P. \& Völlink, T. (2012). Tackling cyberbullying: Review of empirical evidence regarding successful responses by students, parents, and schools. International Journal of Conflict and Violence, 6, (2), 283-293.

Perren, S. \& Gutzwiller-Helfenfinger, E. (2012). Cyberbullying and traditional bullying in adolescence: Differential roles of moral disengagement, moral emotions, and moral values. European Journal of Developmental Psychology, 9, (2), 195-209.

Petermann, F. \& von Marées, N. (2013). Cyber-Mobbing: Eine Bestandsaufnahme. Kindheit und Entwicklung, 22, (3), 145-154.

Pfetsch, J., Mohr, S. \& Ittel, A. (2012). Cyber-Mobbing. Formen, Funktionen und Auswirkungen im Leben Jugendlicher. AJS-Informationen, 48, (2), 4-7.

Pfetsch, J., Mohr, S. \& Ittel, A. (2014). Prävention und Intervention von Online-Aggressionen: Wie wirksam sind Maßnahmen, die sich spezifisch gegen Cybermobbing richten? In S. Pieschl \& T. Porsch (Hrsg.), Neue Medien und deren Schatten. Mediennutzung, Medienwirkung, Medienkompetenz (S. 277-300). Göttingen: Hogrefe Verlag. 
Pieschl, S. \& Porsch, T. (2012). Schluss mit Cybermobbing! Das Trainings- und Präventionsprogramm «Surf-Fair». Weinheim: Beltz.

Pieschl, S. \& Porsch, T. (2014). Cybermobbing - mehr als «Ärgern im Internet». In S. Pieschl $\&$ T. Porsch (Hrsg.), Neue Medien und deren Schatten. Mediennutzung, Medienwirkung, Medienkompetenz (S. 133-158). Göttingen: Hogrefe Verlag.

Pieschl, S., Porsch, T., Kahl, T. \& Klockenbusch, R. (2013). Relevant dimensions of cyberbullying - results from two experimental studies. Journal of Applied Developmental Psychology, 34, 241-252.

Porsch, T. \& Pieschl, S. (2014). Cybermobbing unter deutschen Schülerinnen und Schülern: Eine repräsentative Studie zu Prävalenz, Folgen und Risikofaktoren. Diskurs Kindheitsund Jugendforschung, 9, (1), 7-22.

Reinecke, J. \& Pöge, A. (2010). Strukturgleichungsmodelle. In C. Wolf \& H. Best (Hrsg.), Handbuch der sozialwissenschaftlichen Datenanalyse (S. 775-804). Wiesbaden: VS Verlag für Sozialwissenschaften.

Riebel, J., Jäger, R. S. \& Fischer, U. (2009). Cyberbullying in Germany - an exploration of prevalence, overlapping with real life bullying and coping strategies. Psychology Science, 51, (3), 298-314.

Schubarth, W. (2012). Gewalt und Mobbing an Schulen: Möglichkeiten der Prävention und Intervention. Stuttgart: Kohlhammer.

Selman, R. L. (1980). The growth of interpersonal understanding. New York, NY: Academic Press.

Selman, R. L. (2003). The promotion of social awareness: Powerful lessons from the partnership of developmental theory and classroom practice. New York, NY: Russell Sage.

Slonje, R. \& Smith, P. K. (2008). Cyberbullying: Another main type of bullying? Scandinavian Journal of Psychology, 49, (2), 147-154.

Slonje, R., Smith, P. K. \& Frisén, A. (2013). The nature of cyberbullying, and strategies for prevention. Computers in Human Behavior, 29, (1), 26-32.

Smith, P. K., Mahdavi, J., Carvalho, M., Fisher, S., Russell, S. \& Tippett, N. (2008). Cyberbullying. Its nature and impact in secondary school pupils. Journal of Child Psychology and Psychiatry, 49, (4), 376-385.

Sticca, F., Perren, S., Ruggieri, S. \& Alsaker, F. (2013). Longitudinal risk factors for cyberbullying in adolescence. Journal of Community and Applied Social Psychology, 23, (1), 52-67.

Teuschel, P. (2010). Mobbing: Dynamik - Verlauf - gesundheitliche und soziale Folgen. Stuttgart: Schattauer.

Voskamp, F. \& Kipker, D.-K. (2013). Virtueller Pranger Internet. Datenschutz und Datensicherheit, 37, (12), 787-790.

Wachs, S., Hess, M., Scheithauer, H. \& Schubarth, W. (2016). Mobbing an Schulen: Erkennen - Handeln - Vorbeugen. Stuttgart: Kohlhammer.

Wachs, S. \& Wolf, K. D. (2011). Über den Zusammenhang von Bullying und Cyberbullying. Erste Ergebnisse einer Selbstberichtsstudie. Praxis der Kinderpsychologie und Kinderpsychiatrie, 60, (9), 735-744.

Waller, G., Willemse, I., Genner, S., Suter, L. \& Süss, D. (2016). JAMES - Jugend, Aktivitäten, Medien - Erhebung Schweiz. Zürich: Zürcher Hochschule für Angewandte Wissenschaften.

Weinstein, E. \& Selman, R. L. (2014). Digital stress: Adolescents' personal accounts. New Media \& Society, 18, (3), 391-409.

Willard, N. E. (2007). Cyberbullying and cyberthreats. Responding to the challenge of online social aggression, threats, and distress. Champaign, IL: Research Press.

Schlagworte: Cybermobbing, Einstellungen, Messmodell 


\section{Insultes, rumeurs, dénigrement dans l'espace virtuel: un modèle de mesure de l'attitude d'élèves envers le cybermobbing}

\section{Résumé}

Dans une époque imprégnée par les médias sociaux, le cybermobbing renvoie à un phénomène problématique qui ne peut pas être sous-estimé. La présente contribution s'y intéresse tout particulièrement, tentant de savoir (1) si les attitudes à l'égard du cybermobbing se laissent caractériser empiriquement et (2) quelles attitudes montrent les élèves à son égard. L'échantillon de l'analyse présentée provient du module national de l'étude IEA / ICILS pour la Suisse. Il comprend des élèves de $8 \mathrm{e}$ ainsi que des jeunes ayant participé à une étude complémentaire menée en Suisse orientale. Un nouvel instrument permettant de saisir les attitudes des élèves à l'égard du cybermobbing a été développé et est présenté ici. Les deux bases de données de cette étude confirment confirment un modèle de facteur $g$ fondé théoriquement. Les résultats montrent que les élèves, dans une grande majorité, ont une attitude négative à l'égard du cybermobbing.

Mots-clés: Cybermobbing, attitudes, modèle de facteur g

\section{Insulti, pettegolezzi e denigrazione nello spazio virtuale: modello di misurazione delle attitudini verso il cyberbullismo degli studenti}

\section{Riassunto}

Il cyberbullismo pone un problema che non dovrebbe essere sottovalutato in questa epoca dei social media. Questo saggio si chiede (1) se gli atteggiamenti nei confronti del cyberbullismo possono essere compresi empiricamente e (2) quali atteggiamenti gli allievi e le allieve mostrano nei confronti del cyberbullismo. Il campione rappresentativo della presente ricerca proviene dallo studio IEA ICILS in Svizzera (modulo nazionale) e comprende sia gli alunni e le alunne dell $>$ ottava classe (scuola media) che i e le partecipanti ad uno studio supplementare sui giovani della Svizzera orientale. Per rilevare l'atteggiamento nei confronti del cyberbullismo è stato recentemente sviluppato uno strumento, che viene presentato in questo saggio. I dati raccolti da entrambe le fonti confermano un modello del fattore $g$ motivato teoricamente. I risultati mostrano che la netta maggioranza degli allievi e delle allieve ha un atteggiamento negativo nei confronti del cyberbullismo.

Parole chiave: Cyberbullismo, atteggiamenti, modello del fattore g 


\section{Insulting, Spreading Rumors and Denigrating in Virtual Space: Measurement Model for the Attitudes Towards Cyberbullying of Students}

\section{Summary}

Cyberbullying is a challenge in times of social media. This paper poses the following two questions: (1) How can attitudes towards cyberbullying be empirically measured, and (2) what attitudes do students show towards cyberbullying. Two samples serve to answer these research questions: the first survey is part of the IEA study ICILS in Switzerland (national module) which includes eighth grade students. The second survey derives from a study carried out in Eastern Switzerland with young people from age 11 to 16 . Construct validity of a newly developed instrument for measuring students' attitudes towards cyberbullying is presented in this paper. Both data sets confirm a theoretically justified g-factor model. The results show that a clear majority of students is opposed to cyberbullying.

Keywords: Cyberbullying, attitudes, measurement model 